\title{
Pulmonary rehabilitation in COPD: a case study
}

\begin{abstract}
Dyspnea is the main symptom in patients with chronic obstructive pulmonary disease (COPD) and bronchodilators are the principal pharmacological treatment. We present a case of a patient treated in a pulmonary rehabilitation program who improved his functional capacity and quality of life. We will discuss the impact of a pulmonary rehabilitation program on cardiopulmonary exercise testing with and without broncodilator.
\end{abstract}

Keywords: COPD, pulmonary rehabilitation, physical training, rehabilitation
Volume 3 Issue I - 2018

\author{
Paulo José Zimermann Teixeira, ${ }^{1,2}$ Carine \\ Lumi $^{2}$ \\ 'Hospital Pavilhão Pereira Filho, Santa Casa de Misericórdia de \\ Porto Alegre, Brazil \\ ${ }^{2}$ Health Sciences Post Graduation Course, Universidade Federal \\ de Ciências da Saúde de Porto Alegre Brazil
}

\begin{abstract}
Correspondence: Paulo José Zimermann Teixeira, Health Sciences Post Graduation Course, Universidade Federal de Ciências da Saúde de Porto Alegre, Brazil, Email paulozt@ufcspa.edu.br
\end{abstract}

Received: Decenber 31, 2017 | Published: February 16, 2018

\section{Introduction}

A 70-year-old male patient, who worked as a bricklayer, active smoker, 40 cigarettes/day for 60 years. About five years ago he began presenting with dyspnea when performing moderate efforts and productive cough with sputum, usually in the morning. During the last year he was not hospitalized. The patient was on formoterol $12 \mu \mathrm{g}$ three times a day and mometasone $400 \mu \mathrm{g}$, continuously. The patient's height and body mass index (BMI) are $1.58 \mathrm{~cm}$ and $22.3 \mathrm{~kg} /$ $\mathrm{m}^{2}$, respectively. His blood pressure was $140 / 80 \mathrm{mmHg}$, heart rate (HR) 64bpm, respiratory rate (RR) 20rpm and peripheral oxygen saturation $\left(\mathrm{SpO}_{2}\right)$ at rest $95 \%$. Pulmonary auscultation revealed a diffusely reduced vesicular murmur and no alterations were found in cardiac auscultation.

The patient was submitted to a pulmonary function test with the following results after the administration of the bronchodilator: FEV1 $0.92(39.2 \%)$, FVC 1.79(59.2\%) and FEV1/FVC 51.4(68.7\%). In the 6-minute walk test (6MWT), the patient went through a total of 487.4 meters, without significant variations in heart rate and peripheral oxygen saturation. The quality of life assessment was done using the Saint George's Respiratory Questionnaire (SGRQ). After the initial assessment, the patient was included in the pulmonary rehabilitation program (PRP). The program lasted three months and consisted of three weekly training sessions, one weekly educational session and one weekly meeting with the psychology support group. During the PRP, the patient was instructed to maintain the use of any medications he had been taking previously. The CPET was performed using a protocol for incremental submaximal treadmill with incline, with total incremental time ranging from 8 to 12 minutes.

\section{Results and discussion}

The patient went through a total of 36 physical training sessions over a period of three months. The results corresponding to the CPET before and after the PRP and the use of the long-acting bronchodilator are described in Table 1. Exercise tolerance time was significantly higher in the post-PRP CPET, but the patient had stopped using the bronchodilator on his own. After the long-acting bronchodilator was re-introduced, the increase in time was even greater in relation to the pre-PRP time. In the initial CPET, the total lung capacity was $470 \mathrm{ml}$, whereas after the test there was an increase to $530 \mathrm{ml}$ and $610 \mathrm{ml}$ after use of the long-acting bronchodilator. However, it was observed that the perception of dyspnea was lower at $2 \mathrm{~min}$ and $14 \mathrm{~min}$ of exercise according to the Borg scale.

Table I Results of the CPET

\begin{tabular}{llll}
\hline Variables & CPET pre PRP & CPET post PRP & CPET after 2 weeks using a long-acting bronchodilator \\
\hline Limit time(min:s) & $2: 38$ & $13: 50$ & $16: 11$ \\
BORG isotime (D/MI) & $8-\mathrm{Jul}$ & $3 / 0,5$ & $3 / 0,5$ \\
$2 \mathrm{~min}$ & & \\
I4 min & & $2-\mathrm{Sep}$ & $6 / 0,5$ \\
BORG peak (D/MI) & 2 -Aug & $9-\mathrm{Feb}$ & $7 / 0,5$ \\
TLC resting & 2,12 & 1,97 & 2,19 \\
TLC peak & 1,65 & 1,44 & 1,58 \\
SGRQ total & 46 & 27 & - \\
\hline
\end{tabular}

TLC, total lung capacity; SGRQ, saint george's respiratory questionnaire 
The perception of the degree of effort in the lower limbs was a limiting factor for the execution of the initial test. Initially, the patient considered the effort to be very intense. The increase in exercise tolerance occurred with a reduced perception of lower limb effort by the patient and after the PRP there was a reduction in the SGRQ score, demonstrating an improvement in the perception of quality of life. Exercising greatly improves the response to exercise in individuals with COPD mainly through a reduction in lower-limb fatigue. ${ }^{1}$

Studies have shown that although exercise has no effect on lung function, it may reduce the lactate threshold and the production of carbon dioxide $\left(\mathrm{VCO}_{2}\right)$ by improving exercise response. ${ }^{2}$

Changes in pulmonary mechanics associated with exercise are related to the cardiovascular response to exercise. Dynamic hyperinflation (DH) during exercise increases intrathoracic pressure and consequently reduces cardiac preload by reducing venous return and left ventricular volume, and it is likely that in patients with COPD an improvement in ventilation is associated with increased volume during the exercise, consequently enabling more efficient exercise over a longer period of time, as observed in our study. ${ }^{3}$

In addition, exercise in COPD patients without cachexia can reduce type IIb fibers and increase the proportion of type I fibers, requiring less muscle oxygen, resulting in savings of oxygen and carbon dioxide after PRP, which in turn could induce carbon dioxide elimination. ${ }^{4}$ Several mechanisms are involved in improvements induced by the PRP. Current guidelines recommend that pulmonary rehabilitation complement standard pharmacological therapy in moderate to severe COPD patients, through established improvements in quality of life, dyspnea, and functional capacity, reinforcing the need for proactive behaviors that benefit these patients.

\section{Acknoweledgements}

None.

\section{Conflict of interest}

The author declares no conflict of interest.

\section{References}

1. Albuquerque ALP, Quaranta M, Chakrabarti B, et al. Exercise performance and differences in physiological response to pulmonary rehabilitation in severe chronic obstructive pulmonary disease with hyperinflation. J Bras Pneumol. 2016;42(2):121-129.

2. Neder JÁ, Berton DC, Muller PT, et al. Ventilatory inefficiency and exertional dyspnea in early chronic obstructive pulmonary disease. Ann Am Thorac Soc. 2017;14(1):22-29.

3. Ramponi S, Tzani P, Aiello M, et al. Pulmonary rehabilitation improves cardiovascular response to exercise in COPD. Respiration. 2013;86(1):17-24.

4. Miki K, Maekura R, Kitada S, et al. Pulmonary rehabilitation for COPD improves exercise time rather than exercise tolerance: effects and mechanisms. Int J Chron Obstruct Pulmon Dis. 2017;3(12):1061-1070. 